\title{
CONCRETO HIDRÁULICO MODIFICADO CON SÍLICE OBTENIDA DE LA CASCARILLA DEL ARROZ
}

\section{CONCRETE HYDRAULIC MODIFIED WITH SILICA OBTAINED OF THE RICE HUSK}

Nelson Ricardo Camargo Pérez', Carlos Hernando Higuera Sandoval²

Fecha de recepción: 6 de junio de 2016

Fecha de revisión: 19 de agosto de 2016

Fecha de aprobación: 6 de octubre de 2016

Referencia: N. R. Camargo Pérez, C. H. Higuera Sandoval (2017). Concreto hidráulico modificado con sílice obtenida de la cascarilla del arroz. Ciencia e Ingeniería Neogranadina, 27 (1), pp. 91-109, DOl: http://dx.doi.org/10.18359/rcin.1907

\section{RESUMEN}

El cemento es una de las materias primas en la producción del concreto hidráulico, pero su producción es considerada como uno de los procesos más contaminantes en nuestro planeta. El proyecto de investigación analiza el comportamiento mecánico, físico y químico de una mezcla de concreto hidráulico modificado con sílice obtenida por la incineración de cascarilla de arroz. La metodología utilizada fue un diseño experimental que se trabajó con cemento Holcim M1 Concretera, se utilizó como muestra patrón un diseño de mezcla de concreto hidráulico para una resistencia a la compresión de $350 \mathrm{Kg} / \mathrm{cm}^{2}$, y resistencia a la flexión de $42 \mathrm{Kg} / \mathrm{cm}^{2}$. La modificación se realizó mediante la sustitución del cemento por ceniza de cascarilla de arroz (sílice), en proporciones del 5\%, 15\% y 30\%. Los resultados muestran la viabilidad para el porcentaje

\footnotetext{
1. Ingeniero en Transportes y Vías de la Universidad Pedagógica y Tecnológica de Colombia, Especialista en Diseño, Construcción y Conservación de Vías de la Escuela Colombiana de Ingeniería, Especialista en Infraestructura Vial de la Universidad Pedagógica y Tecnológica de Colombia, Estudiante Maestría en Ingeniería con énfasis en Infraestructura Vial de la Universidad Pedagógica y Tecnológica de Colombia, Miembro del grupo de investigación GRINFRAVIAL, Tunja - Colombia, nelson.camargo01@uptc.edu.co

2. Ingeniero en Transportes y Vías de la Universidad Pedagógica y Tecnológica de Colombia, Especialista en Vías Terrestres de la Universidad del Cauca, Especialista en Carreteras de la Universidad Politécnica de Madrid - España, Especialista en Transportes Terrestres de la Universidad Politécnica de Madrid - España, Magíster en Vías Terrestres de la Universidad del Cauca, Profesor Titular de la Escuela Transporte y Vías Facultad de Ingeniería, Universidad Pedagógica y Tecnológica de Colombia, Director Grupo de Investigación y Desarrollo en Infraestructura Vial - GRINFRAVIAL - Categoría C, Tunja - Colombia, carlos.higuera@uptc.edu.co
} 
de sustitución de 5\% en la resistencia a la compresión, la tracción indirecta y a la flexión, por lo tanto, hay una viabilidad técnica para aplicar este procedimiento.

Palabras clave: cascarilla del arroz, concreto hidráulico, pavimento rígido, puzolana, sílice.

\section{ABSTRACT}

The cement is one of the raw materials in the production of the hydraulic concrete, but its production is considered one of the most polluting processes on our planet. The research project analyzes the mechanical, physical and chemical behavior of a mix of hydraulic concrete modified with silica obtained from the incineration of rice husk. The used methodology was an experimental design that was worked with cement Holcim M1 Concretera, it was used as pattern sample, a mix design of hydraulic concrete for a compressive strength of $350 \mathrm{Kg} / \mathrm{cm}^{2}$, and flexural strength of $42 \mathrm{Kg} / \mathrm{cm}^{2}$. The modification was made by replacing the cement by ash rice husk (silica), in proportions of $5 \%, 15 \%$ and $30 \%$. The results show viability for the replacement percentage of $5 \%$ in the compressive strength, indirect strain and flexural, therefore there is a technical feasibility to apply this procedure.

Keywords: husk of rice, hydraulic concrete, rigid pavement, pozzolan, silica.

\section{INTRODUCCIÓN}

El cemento es una de las materias primas en la construcción de las obras civiles en todo el mundo pero su producción es considerada uno de los procesos más contaminantes que existen en nuestro planeta [1], de allí nace la idea de encontrar nuevos materiales que puedan reemplazar las propiedades mecánicas que este aporta particularmente al concreto hidráulico en los pavimentos rígidos [2-5].

Se han realizado investigaciones en diferentes países con residuos agroindustriales como sustituto parcial del cemento para la fabricación de mampuestos no estructurales [6-7], morteros [8-11] y concretos [12-16] con resultados prometedores para la ingeniería de materiales.
Actualmente, la producción de arroz y a su vez de cascarilla de arroz en Colombia ha venido presentando un aumento considerable, especialmente en la región de la Orinoquia, caso particular en el departamento de Casanare, donde se cosecharon 426.580 toneladas en el segundo semestre del año 2013 y 423.359 toneladas en el segundo semestre del año 2014 [17]. La cascarilla del arroz no tiene una disposición final ni uso estipulado y está siendo desechada en campos abiertos donde se incinera, generando contaminación al medio ambiente.

La cascarilla de arroz está compuesta básicamente por sílice amorfa, que mediante un proceso térmico se puede obtener con un grado de pureza significativo [4, 18-19] en forma de ceniza, tratando de darle un 
aprovechamiento y valor agregado, se propone realizar una sustitución de cemento por ceniza obtenida en la incineración de cascarilla del arroz en diferentes porcentajes $[4,13-15,20]$ en un diseño de mezcla de concreto hidráulico para pavimento.

En otras investigaciones, se ha utilizado la ceniza de cascarilla del arroz obtenida mediante un tratamiento químico y térmico como sustituto parcial del cemento en mezclas de concreto hidráulico, encontrándose incremento en la resistencia a flexión [14, 15, 23], también se ha utilizado la ceniza de cascarilla de arroz como aditivo en morteros, obteniéndose morteros ligeros pero de baja resistencia mecánica $[1,5,9]$.

En el desarrollo de este proyecto se trabajó una metodología experimental, realizando diferentes ensayos de laboratorio para conocer el comportamiento y las propiedades mecánicas del concreto hidráulico, modificado con el $5 \%, 15 \%$ y $30 \%$ de sustitución de cemento por ceniza, teniendo en cuenta la norma INVIAS 2013 para ensayos sobre concretos hidráulicos para pavimentos. Adicionalmente, se emplean técnicas de análisis como la Difracción de Rayos X (DRX) y el Microscopio Electrónico de Barrido (MEB) [20-23], para conocer la composición mineralógica y morfología de la ceniza, el cemento utilizado y los especímenes fabricados para los diferentes tipos de ensayos a compresión, flexión y tracción indirecta.

\section{MATERIALES Y MÉTODOS}

\subsection{Materiales}

Los materiales empleados para el desarrollo del proyecto fueron los que comúnmente se usan en la fabricación del concreto hidráulico, como arena, grava y cemento, adicionalmente se utilizó cascarilla del arroz como material base de la investigación.

Para el suministro de los materiales pétreos (arena y grava) se realizó un sondeo de las fuentes de material existentes y habilitadas que estuvieran ubicadas dentro de la ciudad de Yopal; teniendo en cuenta la distancia de acarreo, se seleccionó la fuente de materiales "CRASURCA S.A.", el tipo de cemento utilizado fue Holcim M1 Concretera, por ser el más usado en las plantas concreteras, este se adquirió en la planta de PETRORIENTE S.A.S. y la cascarilla de arroz se obtuvo de la planta procesadora de arroz DiCorp, ubicada en el kilómetro 5 vía Yopal - Morichal, margen derecha, en la modalidad de prensado.

\subsection{Métodos}

\subsubsection{Caracterización de materiales}

La caracterización de los materiales pétreos se realizó de acuerdo con las normas de ensayos de materiales para carreteras INVIAS 2013, cuya finalidad es garantizar la calidad de los materiales utilizados, cada material tiene una serie de ensayos (Tabla 1) y unos resultados esperados para cumplir a cabalidad con las especificaciones.

La cascarilla del arroz se sometió a un proceso de incineración a una temperatura de $+/-800^{\circ} \mathrm{C}$ por medio de un horno durante un periodo de una hora, teniendo como principio básico que a medida que la temperatura es más alta elimina por completo cualquier tipo de resto orgánico presente en la cascarilla del arroz y esto garantiza mejores resultados en la elaboración del concreto hidráulico [4], sin embargo, 
Tabla 1. Ensayos a los agregados propuestos por la norma INVIAS 2013

\begin{tabular}{|c|c|c|}
\hline Ítem & Material & Ensayo \\
\hline \multirow{3}{*}{1} & \multirow{3}{*}{$\begin{array}{l}\text { Material granular } \\
\text { fino (arena de } \\
\text { trituración) }\end{array}$} & I.N.V. E-133-13 Equivalente de arena de suelos y agregados finos \\
\hline & & I.N.V. E-213-13 Análisis granulométrico de los agregados gruesos y finos \\
\hline & & $\begin{array}{l}\text { I.N.V. E-222-13 Densidad, densidad relativa (gravedad especifica) y absorción del } \\
\text { agregado fino }\end{array}$ \\
\hline \multirow{5}{*}{2} & \multirow{5}{*}{$\begin{array}{c}\text { Material granular } \\
\text { grueso (triturado de } \\
3 / 4^{\prime \prime} \text { ) }\end{array}$} & I.N.V. E-213-13 Análisis granulométrico de los agregados gruesos y finos \\
\hline & & $\begin{array}{l}\text { I.N.V. E-218-13 Resistencia a la degradación de los agregados de tamaños menores } \\
\text { de } 37 \text { mm } 11 / 2 \text { " por medio de la máquina de los ángeles }\end{array}$ \\
\hline & & I.N.V. E-223-13 Densidad, densidad relativa y absorción del agregado grueso \\
\hline & & I.N.V. E-227-13 Porcentaje de las partículas fracturadas en un agregado grueso \\
\hline & & $\begin{array}{l}\text { I.N.V. E-230-13 Índices de aplanamiento y alargamiento de los agregados para } \\
\text { carreteras }\end{array}$ \\
\hline
\end{tabular}

Fuente: Elaboración propia.

cuando la temperatura de incineración es muy alta o el tiempo de residencia es prolongado, la sílice puede perder reactividad.

Como se quiere sustituir cemento por ceniza de la cascarilla del arroz, se determinó que se usaría la ceniza que pasara el tamiz $\mathrm{N}^{\circ}$ $100(0.15 \mathrm{~mm})$, por lo tanto se sometió a un proceso de molienda manual y se tamizó con el fin de homogeneizar el tamaño del grano.

Para la caracterización mineralógica de la ceniza de la cascarilla del arroz se contó con el apoyo del Instituto para la Investigación y la Innovación en Ciencia y Tecnología de Materiales - INCITEMA de la Universidad Pedagógica y Tecnológica de Colombia, para realizar análisis en la máquina de Difracción de Rayos X - DRX y Microscopio Electrónico de Barrido - MEB, para lo cual se tomó una muestra de aproximadamente $20 \mathrm{~g}$ de ceniza debidamente empacada y referenciada.

\subsubsection{Diseño de mezcla}

El diseño de mezcla se genera teniendo en cuenta el manual de diseño de concretos del ingeniero Gerardo Rivera, basándonos en la densidad de los agregados tanto finos como gruesos, la relación agua/cemento y el asentamiento que queremos lograr, todos estos bajo el criterio de la Norma INVIAS 2013.

\subsubsection{Fabricación de especímenes}

De acuerdo al diseño experimental se realizan ensayos de resistencia a compresión, tracción indirecta y flexión, con tres repeticiones para cada ensayo (Tabla 2), en el caso de los ensayos a compresión estos se realizarán a las edades de curado de 7, 14 y 28 días respectivamente, para los ensayos de tracción indirecta y flexión se realizarán a los 28 días de curado. 
Tabla 2. Relación muestras concreto hidráulico con cemento Holcim M1 Concretera

\begin{tabular}{|l|c|c|c|c|c|}
\hline \multicolumn{5}{|c|}{ Concreto $\mathbf{3 4 . 4 7}$ MPa con Cemento Holcim M1 Concretera } \\
\hline \multicolumn{1}{|c|}{ Ensayo } & \multicolumn{1}{c|}{$\begin{array}{c}\text { Tipo } \\
\text { Espécimen }\end{array}$} & $\begin{array}{c}\text { Concreto } \\
\text { Patrón }\end{array}$ & $\begin{array}{c}\text { Concreto con } \\
\text { sustitución } \\
\mathbf{5 \%}\end{array}$ & $\begin{array}{c}\text { Concreto con } \\
\text { sustitución } \\
\mathbf{1 5 \%}\end{array}$ & $\begin{array}{c}\text { Concreto con } \\
\text { sustitución 30\% }\end{array}$ \\
\hline $\begin{array}{l}\text { I.N.V.E-410-13 Resistencia a la } \\
\text { compresión de cilindros de concreto }\end{array}$ & Cilindro & 9 & 9 & 9 & \\
\hline $\begin{array}{l}\text { I.N.V.E-411-13 Ensayo de tracción } \\
\text { por hendimiento (tracción indirecta) } \\
\text { de cilindros de concreto }\end{array}$ & Cilindro & 3 & 3 & 3 & 3 \\
\hline $\begin{array}{l}\text { I.N.V.E-414-13 Resistencia a la } \\
\text { flexión del concreto usando una } \\
\text { viga simplemente apoyada y } \\
\text { cargada en los tercios de la luz libre }\end{array}$ & Vigueta & 3 & 3 & 3 & \\
\hline
\end{tabular}

Fuente: Elaboración propia.

La elaboración de los especímenes de concreto se llevó a cabo en tres jornadas, las variables ambientales de temperatura y humedad relativa estuvieron en condiciones normales.

Durante la elaboración de las muestras de concreto se desarrolló el ensayo de asentamiento del concreto hidráulico Slump (I.N.V.E.-404-13), realizando un ensayo por cada ciclo de mezclado para un total de 8 ensayos, los valores de Slump oscilan entre $25 \mathrm{~mm}$ y $50 \mathrm{~mm}$ parámetros establecidos en la norma INVIAS 2013, presentando buena manejabilidad y consistencia en la mezcla a la hora de tomar los cilindros y las viguetas correspondientes.

\subsubsection{Ensayos de resistencia}

Resistencia a la compresión de cilindros de concreto (I.N.V. E- 410-13). En este ensayo se mide de manera cuantitativa la resistencia que presenta una muestra de concreto cilíndrica al ser comprimida por dos fuerzas iguales ejercidas en sus dos caras trasversales.

Ensayo de tracción por hendimiento (tracción indirecta) de cilindros de concreto (I.N.V E-41113). El ensayo de tracción indirecta se realizó como parámetro de comprobación, teniendo en cuenta que existe una relación entre su valor y el valor de la resistencia a la compresión el cual equivale al $10 \%$ [24].

Resistencia a la flexión del concreto usando una viga simplemente apoyada y cargada en los tercios de la luz libre (I.N.V E-414-13). Este ensayo permite determinar la resistencia a la flexión que presenta un espécimen de concreto cuando se encuentra apoyado en los extremos de su cara longitudinal y es cargado en los tercios de la luz libre entre los apoyos.

El módulo de rotura presenta valores que varían entre un $10 \%$ y $20 \%$ de la resistencia a 
la compresión [24]. Una relación aproximada, que puede utilizarse cuando no se disponga de ensayos a flexión, es la siguiente:

$$
M R=k(R C)^{\frac{1}{2}}
$$

donde:

$M R=$ Módulo de rotura estimado para el concreto $\left(\mathrm{Kg} / \mathrm{cm}^{2}\right)$

$R C=$ Resistencia a la compresión obtenida en el concreto $\left(\mathrm{Kg} / \mathrm{cm}^{2}\right)$

$k=$ Constante que varía normalmente entre 2.0 y 2.7 , para resistencias en $\mathrm{Kg} / \mathrm{cm}^{2}$ a 28 días

Para realizar una comparación del módulo de rotura entre la relación teórica y el resultado de laboratorio se utilizó un valor de $k=2.25$, que de acuerdo a la teoría este valor varía en función de la calidad de los agregados utilizados en la mezcla, de tal forma que reemplazando este valor y la resistencia a la compresión respectiva tenemos:

$$
\begin{gathered}
M R=2.25\left(351.5^{\mathrm{Kg}} \mathrm{cm}^{2}\right)^{\frac{1}{2}} \\
M R=42.18^{\mathrm{Kg}} /_{\mathrm{cm}^{2}} \equiv 4.21 \mathrm{Mpa}
\end{gathered}
$$

\subsubsection{Análisis estadístico de datos}

Se analizó la información resultante por cada variable definida; resistencia a la compresión, resistencia a la tracción indirecta y resistencia a la flexión, se utilizó la estadística para determinar parámetros como el error experimental, media aritmética, grado de significancia.

\section{RESULTADOS Y DISCUSIÓN}

La caracterización que se hizo a los agregados pétreos está consignada en la Tabla 3.

Tabla 3. Caracterización agregados pétreos

\begin{tabular}{|c|l|c|c|}
\hline \multicolumn{1}{|c|}{ Ensayo } & Resultado & $\begin{array}{l}\text { Requisito } \\
\text { INV-2013 }\end{array}$ \\
\hline 1 & $\begin{array}{l}\text { A.N.V.E-133-13 } \\
\text { (Equivalente de } \\
\text { arena, mínimo \%) }\end{array}$ & $61 \%$ & $\geq 60 \%$ \\
\hline 2 & $\begin{array}{l}\text { I.N.V.E-222-13 } \\
\text { (Absorción de agua, } \\
\text { máximo \%) }\end{array}$ & $1.40 \%$ & $\leq 4 \%$ \\
\hline 3 & $\begin{array}{l}\text { AGREGADO GRUESO } \\
\text { (Desgaste en la } \\
\text { máquina de los } \\
\text { ángeles, máximo \%) }\end{array}$ & $37 \%$ & $\leq 40 \%$ \\
\hline 4 & $\begin{array}{l}\text { I.N.V.E-227-13 } \\
\text { (Partículas } \\
\text { fracturadas } \\
\text { mecánicamente, } \\
\text { mínimo \%) }\end{array}$ & $100 \%$ & $\geq 60 \%$ \\
\hline
\end{tabular}

Fuente: Elaboración propia.

Con respecto al análisis granulométrico del agregado fino y grueso cumplen con la gradación requerida, como se puede observar en las Figuras 1 y 2.

El análisis de DRX determinó que en la ceniza están presentes óxidos de silicio tipo cristobalita, cuarzo y un silicato tipo ilita, óxidos de magnesio tipo brusita y carbonatos de calcio tipo calcita (Figura 3 y Tabla 4).

De acuerdo a la Figura 3 y Tabla 4 se tiene un porcentaje de óxido de silicio alto presente 


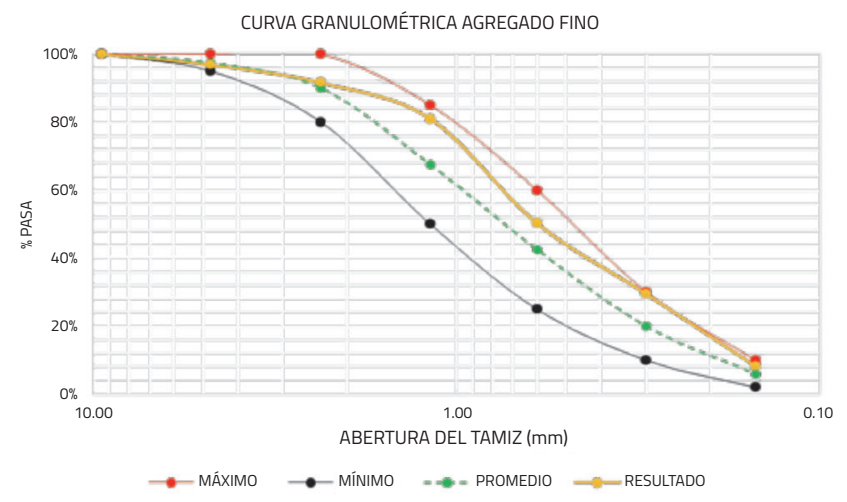

Figura 1. Curva granulométrica agregado fino.

Fuente: Elaboración propia.

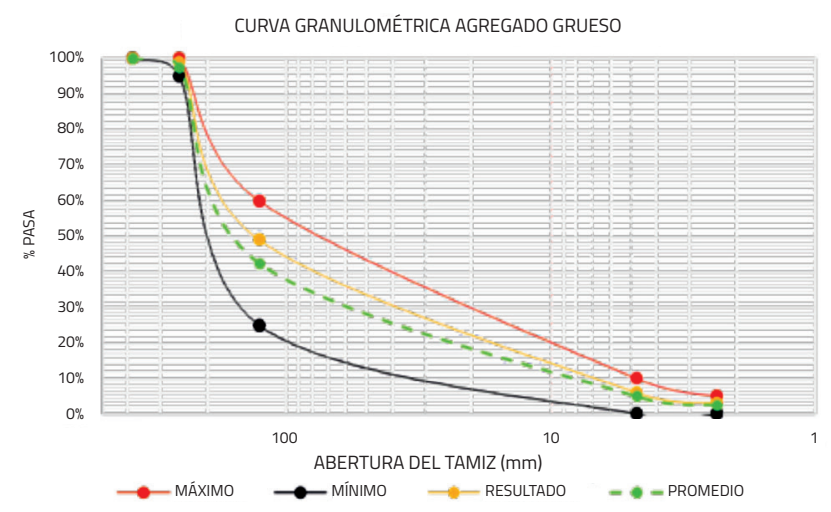

Figura 2. Curva granulométrica agregado grueso.

Fuente: Elaboración propia.

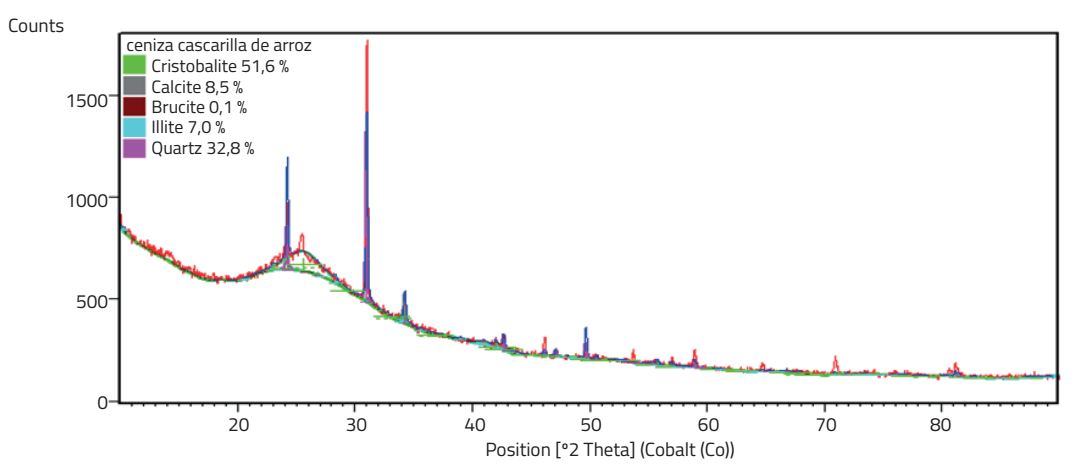

Figura 3. Especies presentes en la ceniza.

Fuente: INCITEMA. 
Tabla 4. Resultados del ensayo DRX.

\begin{tabular}{|c|c|c|c|c|c|c|c|c|}
\hline No. & Visi... & Ref. Code & Compound $\mathrm{Na}$... & Chemical Formula & Score & Scale ... & Display Co... & Crystal System \\
\hline 1 & $\square$ & COD 96-900-8228 & Cristobalite & Si4.00 08.00 & 14 & 0,088 & Lime & Tetragonal \\
\hline 2 & $\square$ & Cop $96-900-9668$ & Calcite & Ca6.00 C6.00 018.00 & 16 & 0,107 & Gray & Hexagonal \\
\hline 3 & $\square$ & Cod 96-900-2355 & Brucite & $\mathrm{Mg} 1.00 \mathrm{O} 2.00$ & 7 & 0,017 & Mar... & Hexagonal \\
\hline 4 & $\square$ & COD 96-901-3721 & Illite & K2.00 Al4.00 Si 8.00 O24.00 & 11 & 0,020 & Aqua & Monoclinic \\
\hline 5 & $\square$ & CoD 96-901-0147 & Quartz & Si 6.0006 .00 & 16 & 0,602 & Fuc... & Hexagonal \\
\hline
\end{tabular}

\section{Fuente: INCITEMA.}

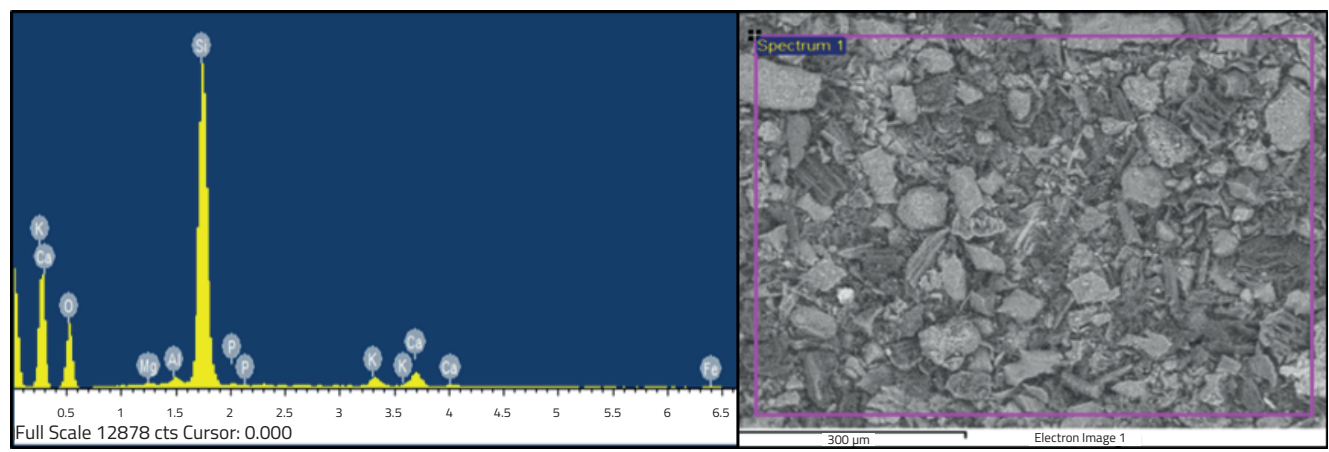

Figura 4. Espectro ceniza cascarilla del arroz.

Fuente: INCITEMA.

en la muestra de la ceniza, el cual equivale al $91.4 \%$ (cristobalita, cuarzo y silicato tipo ilita), lo cual corrobora la información encontrada en la revisión bibliográfica.

Con respecto al análisis de MEB realizado a la ceniza de la cascarilla del arroz se encontró que tiene una morfología laminar (Figura 4) y una presencia alta de sílice.

La composición química de la ceniza de la cascarilla del arroz se presenta en la Tabla 5.

Para la caracterización del cemento la planta de Holcim nos suministró los certificados de calidad que se pueden ver en la Figura 5.
Tabla 5. Caracterización química de la ceniza

\begin{tabular}{|c|c|}
\hline \multicolumn{2}{|c|}{ Análisis Químico (\%) } \\
\hline Descripción & Ceniza cascarilla del arroz \\
\hline $\mathrm{SiO}_{2}$ & 90 \\
\hline $\mathrm{Al}_{2} \mathrm{O}_{3}$ & 0.62 \\
\hline $\mathrm{Fe}_{2} \mathrm{O}_{3}$ & 0.5 \\
\hline $\mathrm{CaO}^{2}$ & 1.23 \\
\hline $\mathrm{MgO}$ & 0.34 \\
\hline $\mathrm{Na}_{2} \mathrm{O}$ & $<0.32$ \\
\hline $\mathrm{K}_{2} \mathrm{O}$ & 2.07 \\
\hline $\mathrm{TiO}_{2}$ & 0.03 \\
\hline Pérdida al fuego & 0.5 \\
\hline
\end{tabular}

Fuente: INCITEMA. 
Información General

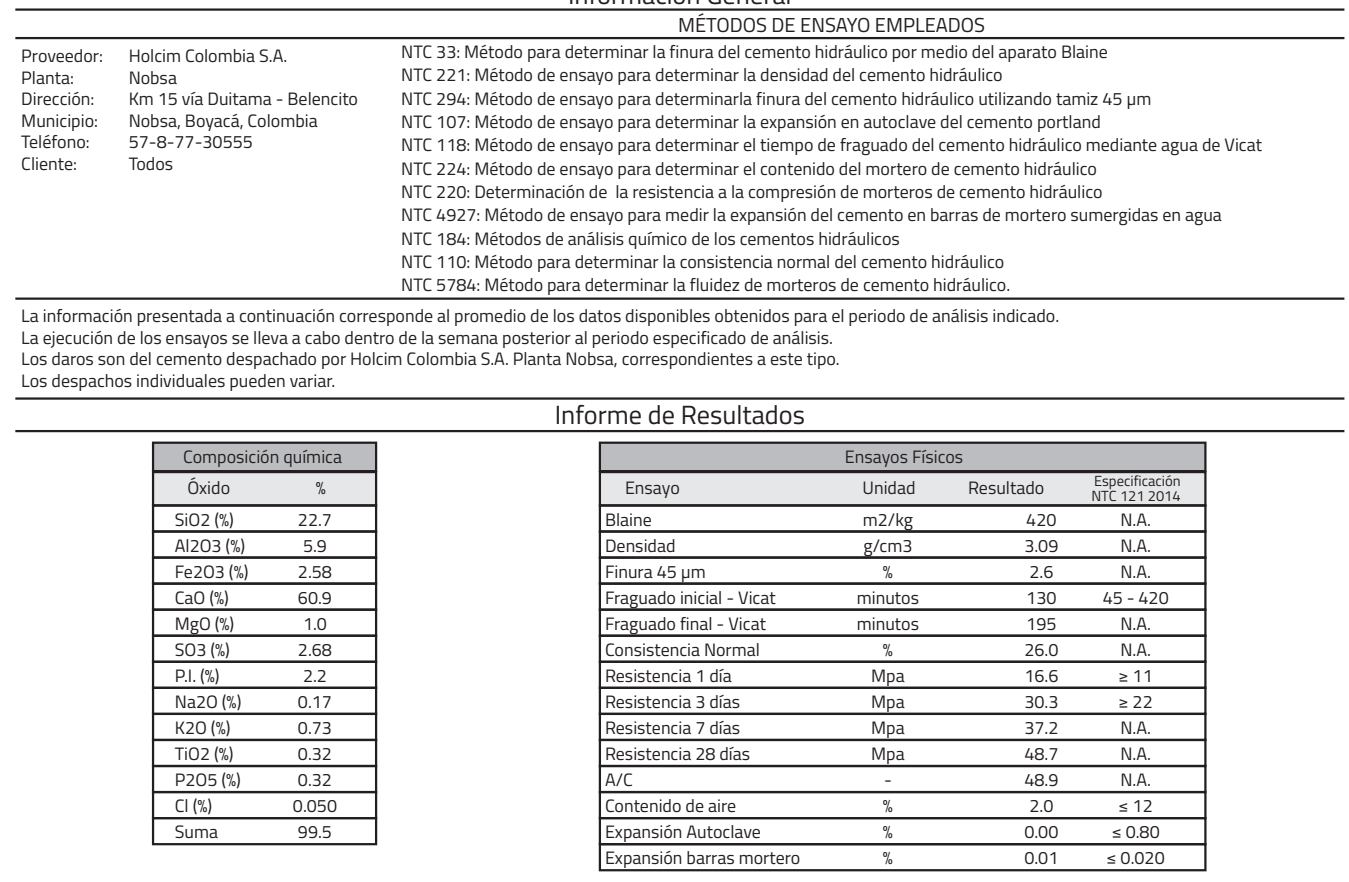

Figura 5. Ficha certificado de calidad cemento Holcim M1 Concretera.

Fuente: Holcim (Colombia) S.A. Planta Nobsa.

En el análisis de DRX para el cemento Holcim M1 Concretera se determinó presencia de minerales de distintos tipos, por parte de los silicatos tenemos presencia de Alita y Harturita dos silicatos de calcio, carbonatos de calcio tipo calcita, minerales de tipo óxido como periclasa y la Brownmillerita (Figura 6 y Tabla 6).

Con respecto al análisis de MEB realizado al cemento M1 Concretera se encontró que tiene una morfología amorfa (Figura 7) y presencia de calcio y sílice.

Con los datos obtenidos en la caracterización de los materiales mediante el desarrollo de los ensayos de laboratorio propuestos, se procede a realizar el diseño mezcla para concreto hidráulico, para el cemento tipo M1 Concretera (Tabla 7) con resistencia a compresión de 34.47 $\mathrm{MPa}$, con el objetivo de lograr un módulo de rotura de 4.2 MPa aproximadamente.

En la Figura 8 se encuentran los resultados de la resistencia a la compresión a los 7 días de curado para los diferentes porcentajes de sustitución, encontrándose que la mayor resistencia es para la muestra con sustitución del $5 \%$ equivalente en promedio a $30.28 \mathrm{MPa}$.

En la Figura 9 se encuentran los resultados de la resistencia a la compresión a los 14 días de curado para los diferentes porcentajes de sustitución, encontrándose que la mayor resistencia es para la muestra patrón equivalente en promedio a $32.84 \mathrm{MPa}$. 


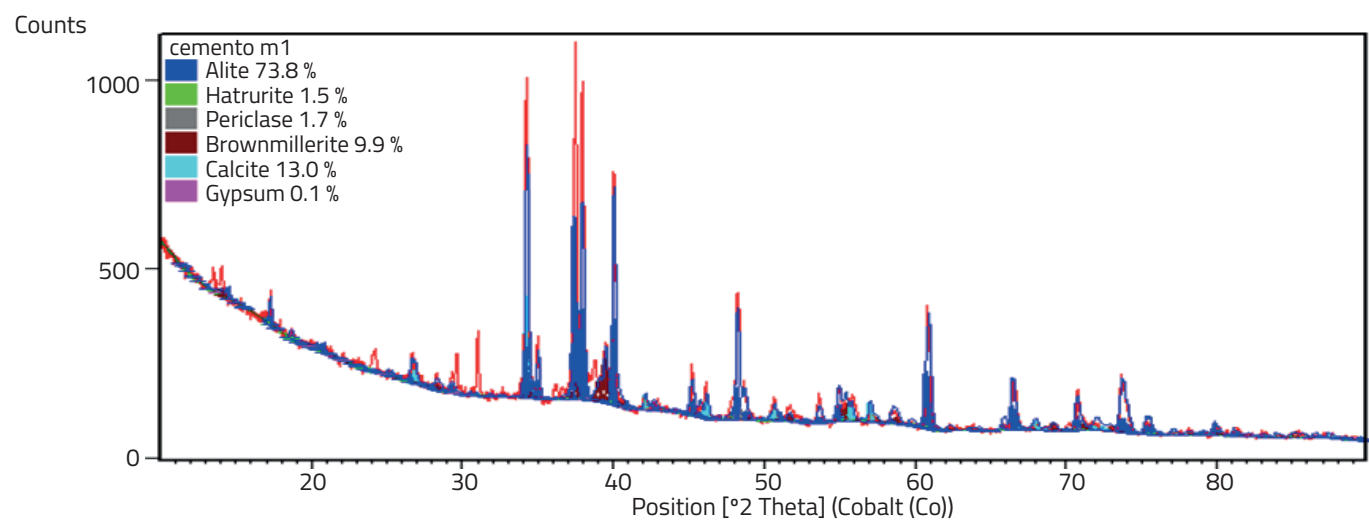

Figura 6. Especies presentes en el cemento Holcim M1 Concretera.

Fuente: INCITEMA.

Tabla 6. Resultados del ensayo DRX cemento Holcim M1 Concretera

\begin{tabular}{|c|c|c|c|c|c|c|c|c|}
\hline No. & Visi... & Ref. Code & Compound $\mathrm{Na}$... & Chemical Formula & Score & Scale ... & Display Co... & Crystal System \\
\hline 1 & $\square$ & Cop 96-901-6126 & Alite & Ca54.00 Si18.00 090.00 & 19 & 0,981 & Blue & Anorthic \\
\hline 2 & $\square$ & Coథ 96-900-9266 & Hatrurite & Ca27.00 Si9.00 O45.00 & 16 & 0,458 & Lime & Hexagonal \\
\hline 3 & $\square$ & Coథ $96-900-6476$ & Periclase & $\mathrm{Mg} 4.0004 .00$ & 12 & 0,248 & Gray & Cubic \\
\hline 4 & $\square$ & CoD 96-901-5721 & Brownmillerite & $\mathrm{Ca} 8.00 \mathrm{Fe} 8.00 \mathrm{O} 20.00$ & 14 & 0,187 & Mar... & Orthorhombic \\
\hline 5 & $\square$ & Coథ $96-901-4878$ & Calcite & Ca6.00 C6.00 018.00 & 13 & 0,292 & Aqua & Hexagonal \\
\hline 6 & 回 & Coథ 96-901-3172 & Gypsum & Ca4.00 S4.00 016.00 At8.00 & 11 & 0,143 & Fuc... & Monoclinic \\
\hline
\end{tabular}

Fuente: INCITEMA.

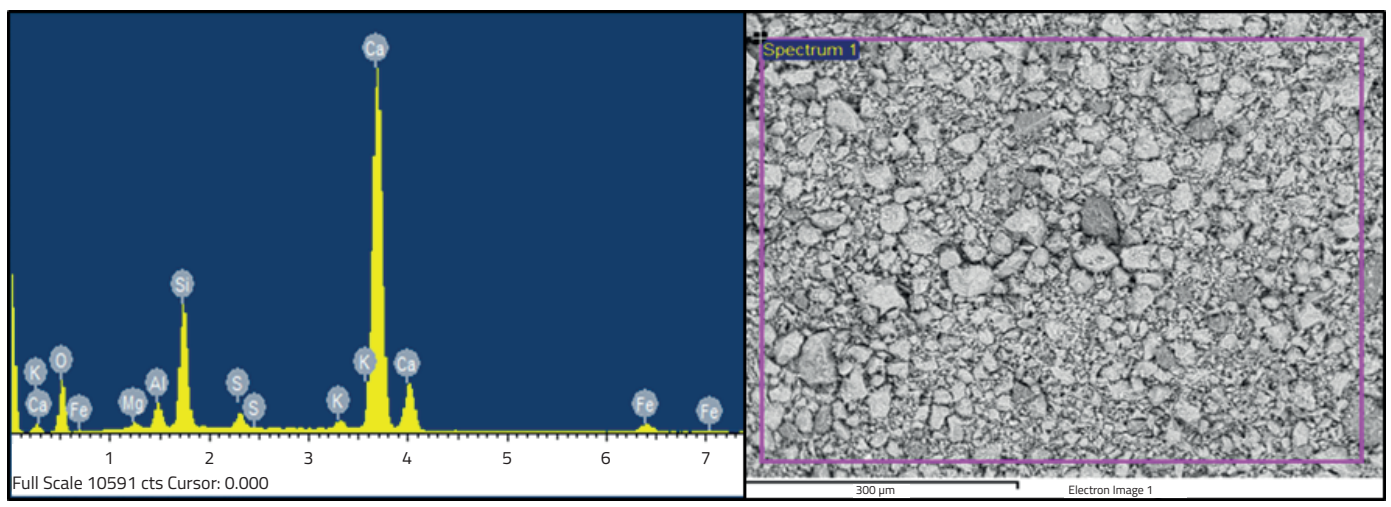

Figura 7. Espectro cemento Holcim M1 Concretera.

Fuente: INCITEMA. 
Tabla 7. Diseño de mezcla para concreto hidráulico con cemento Holcim tipo M1 Concretera.

\begin{tabular}{|c|c|c|c|c|}
\hline Masa de los agregados & \multicolumn{4}{|c|}{$680.21 \mathrm{dm}^{3} * 2.69 \mathrm{Kg} / \mathrm{dm}^{3}=1827.81 \mathrm{Kg} / \mathrm{m}^{3}$} \\
\hline Masa agregado fino & \multicolumn{4}{|c|}{$1828 \mathrm{Kg} / \mathrm{m}^{3} * 38 \%=694.57 \mathrm{Kg} / \mathrm{m}^{3}$} \\
\hline Masa agregado grueso & \multicolumn{4}{|c|}{$1828 \mathrm{Kg} / \mathrm{m}^{3} * 62 \%=1133.24 \mathrm{Kg} / \mathrm{m}^{3}$} \\
\hline \multirow{2}{*}{ Masa materiales ( $\mathrm{kg} / \mathrm{m}^{3}$ de concreto) } & Agua & Cemento & Ag. Fino & Ag. Grueso \\
\hline & 185.00 & 411.11 & 694.57 & 1133.24 \\
\hline Volumen absoluto de materiales ( $\mathrm{dm}^{3} / \mathrm{m}^{3}$ de concreto) & 185.00 & 134.79 & 252.57 & 427.64 \\
\hline Proporción en masa seca & 0.45 & 1.00 & 1.69 & 2.76 \\
\hline
\end{tabular}

Fuente: Elaboración propia.

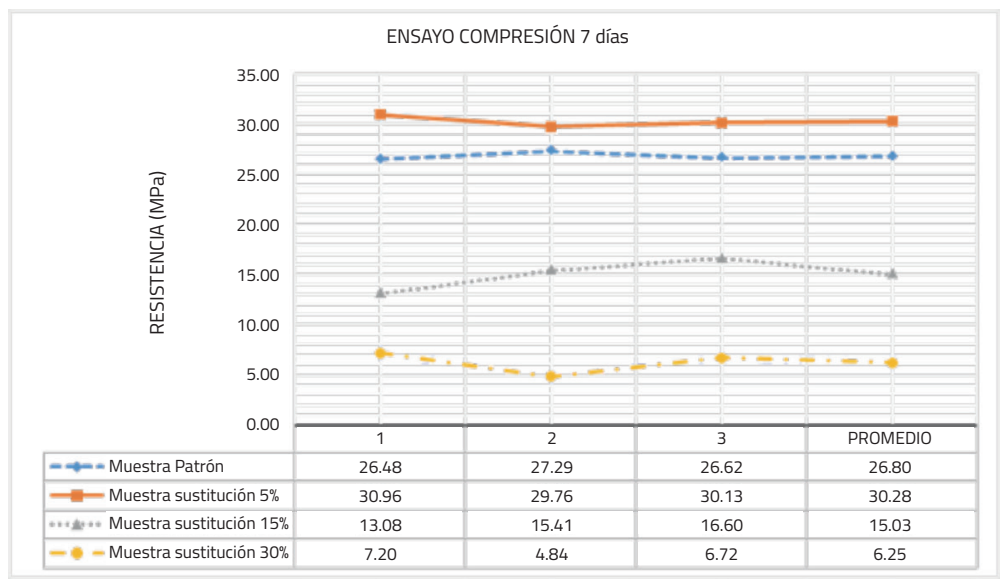

Figura 8. Ensayo compresión 7 días muestras patrón y sustituciones al 5\%, 15\% y 30\%.

Fuente: Elaboración propia.

En la Figura 10 se encuentran los resultados de la resistencia a la compresión a los 28 días de curado para los diferentes porcentajes de sustitución, encontrándose que la mayor resistencia es para la muestra con sustitución del $5 \%$ equivalente en promedio $35.17 \mathrm{MPa}$.

Con este análisis se puede concluir en una primera instancia que las muestras de concreto con sustitución del 5\% presentan una resistencia a la compresión similar a las muestras patrón y mayor que la resistencia de diseño, las muestras con sustitución del $15 \%$ y $30 \%$ por el contrario presentan una disminución significativa de este parámetro con respecto a las muestras patrón y de la resistencia de diseño, por lo tanto no es viable estos porcentajes de sustitución en el diseño de mezcla original. 


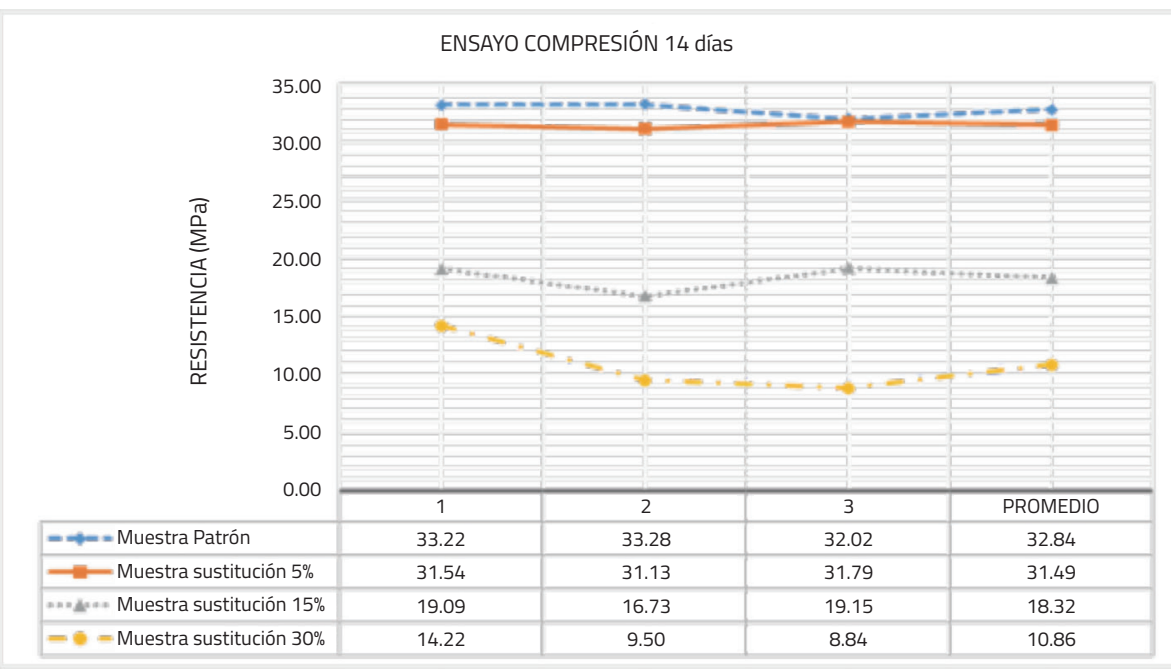

Figura 9. Ensayo compresión 14 días muestras patrón y sustituciones al 5\%, 15\% y 30\%.

Fuente: Elaboración propia.

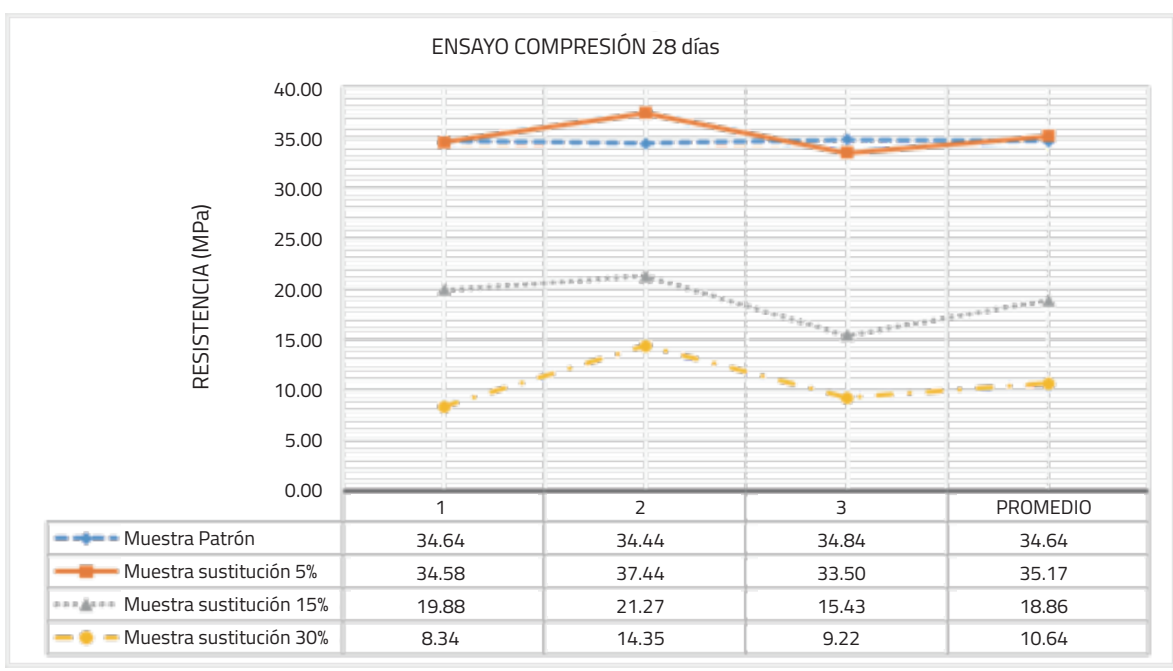

Figura 10. Ensayo compresión 28 días muestras patrón y sustituciones al 5\%, 15\% y 30\%.

Fuente: Elaboración propia.

En la Figura 11 se encuentran los resultados de la resistencia a la tracción indirecta a los 28 días de curado para los diferentes porcentajes de sustitución, encontrándose que la muestra patrón y la muestra con sustitución del 5\% presentan una resistencia en promedio a 3.48 $\mathrm{MPa}$, por lo tanto cumplen con el parámetro de la equivalencia del $10 \%$ del valor de la 


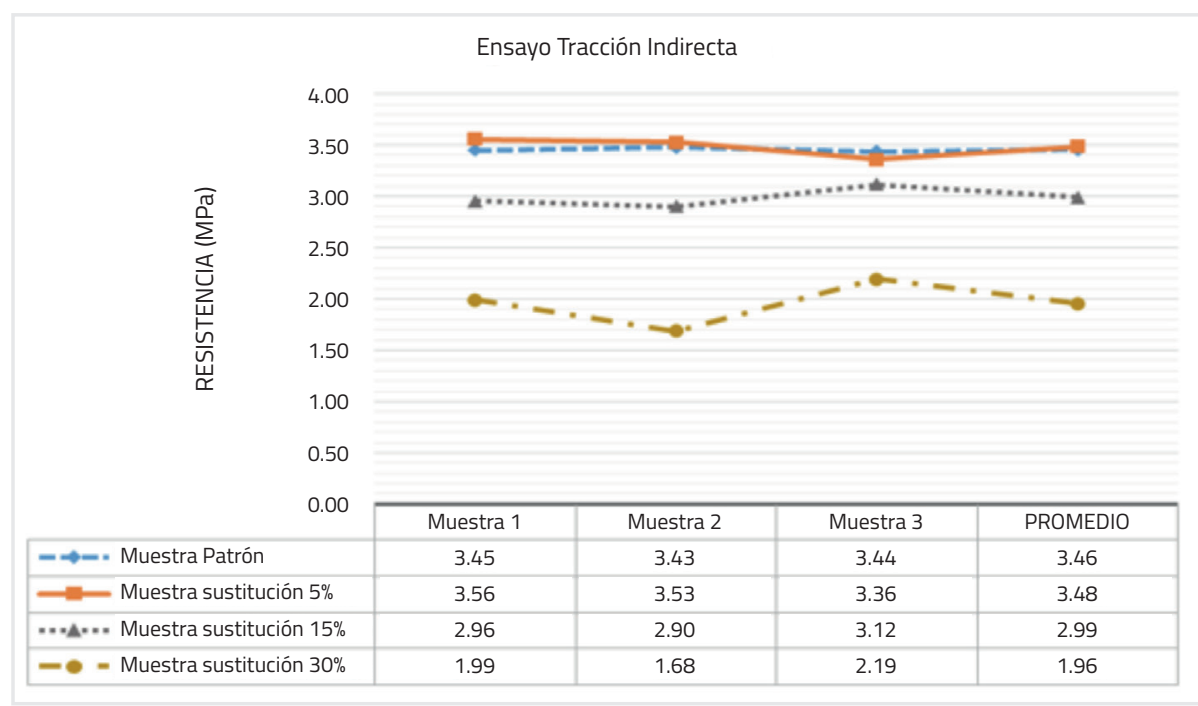

Figura 11. Ensayo tracción indirecta muestras patrón y sustituciones al 5\%, 15\% y 30\%.

Fuente: Elaboración propia.

resistencia de diseño a compresión, para las muestras con sustitución del 15\% y $30 \%$ el valor de la resistencia está por debajo de la relación y por lo tanto no cumplen.

En la Figura 12 se encuentran los resultados de la resistencia a la flexión a los 28 días de curado para los diferentes porcentajes de sustitución, encontrándose que la muestra patrón y la muestra con sustitución del 5\% cumplen con la resistencia de diseño teórica (4.21 MPa), adicionalmente la muestra con sustitución del 5\% presenta un aumento del $9.8 \%$, equivalente a $0.42 \mathrm{MPa}$ con respecto a la resistencia de las muestras patrón; para la muestra con sustitución del 15\% presenta una disminución del $7.12 \%$ con respecto a la resistencia de diseño teórica; para la muestra con sustitución del 30\% el valor de la resistencia disminuye considerablemente con respecto a la resistencia de diseño, por lo tanto no es viable este porcentaje de sustitución en el diseño de mezcla original.

Las muestras de concreto hidráulico con cemento Holcim M1 Concretera se analizaron con DRX, encontrándose para la muestra M1 con sustitución del $0 \%$ presencia del $79.7 \%$ de sílice (Quartz), 18.4\% de calcio (Calcite, Portlandite y Ettringite) y $1.9 \%$ de otras especies; para la muestra M1 con sustitución del $5 \%$ se encontró presencia del $81.4 \%$ de sillice (Quartz), 17.3\% de calcio (Portlandite, Hartrurite, Dolomite, Calcite y Garronite) y $1.3 \%$ de otras especies; para la muestra M1 con sustitución del $15 \%$ se encontró presencia del $62.8 \%$ de sílice (Quartz low y Quartz), 35.4\% de calcio (Portlandite, Calcite y Ettringite) y $1.8 \%$ de otras especies y para la muestra M1 con sustitución del $30 \%$ se halló presencia del 86\% de sílice (Quartz), $11.4 \%$ de calcio (Calcite) y $2.6 \%$ de otras especies (Figura 13). 


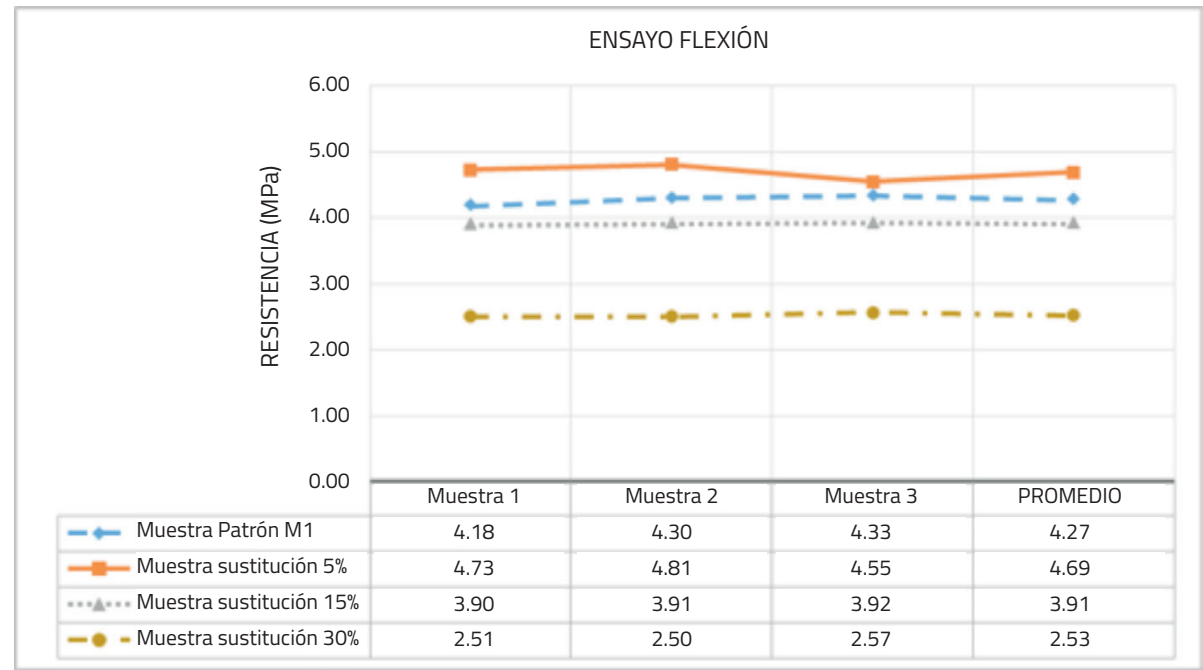

Figura 12. Ensayo a flexión muestras patrón y sustituciones al 5\%, 15\% y 30\%.

Fuente: Elaboración propia
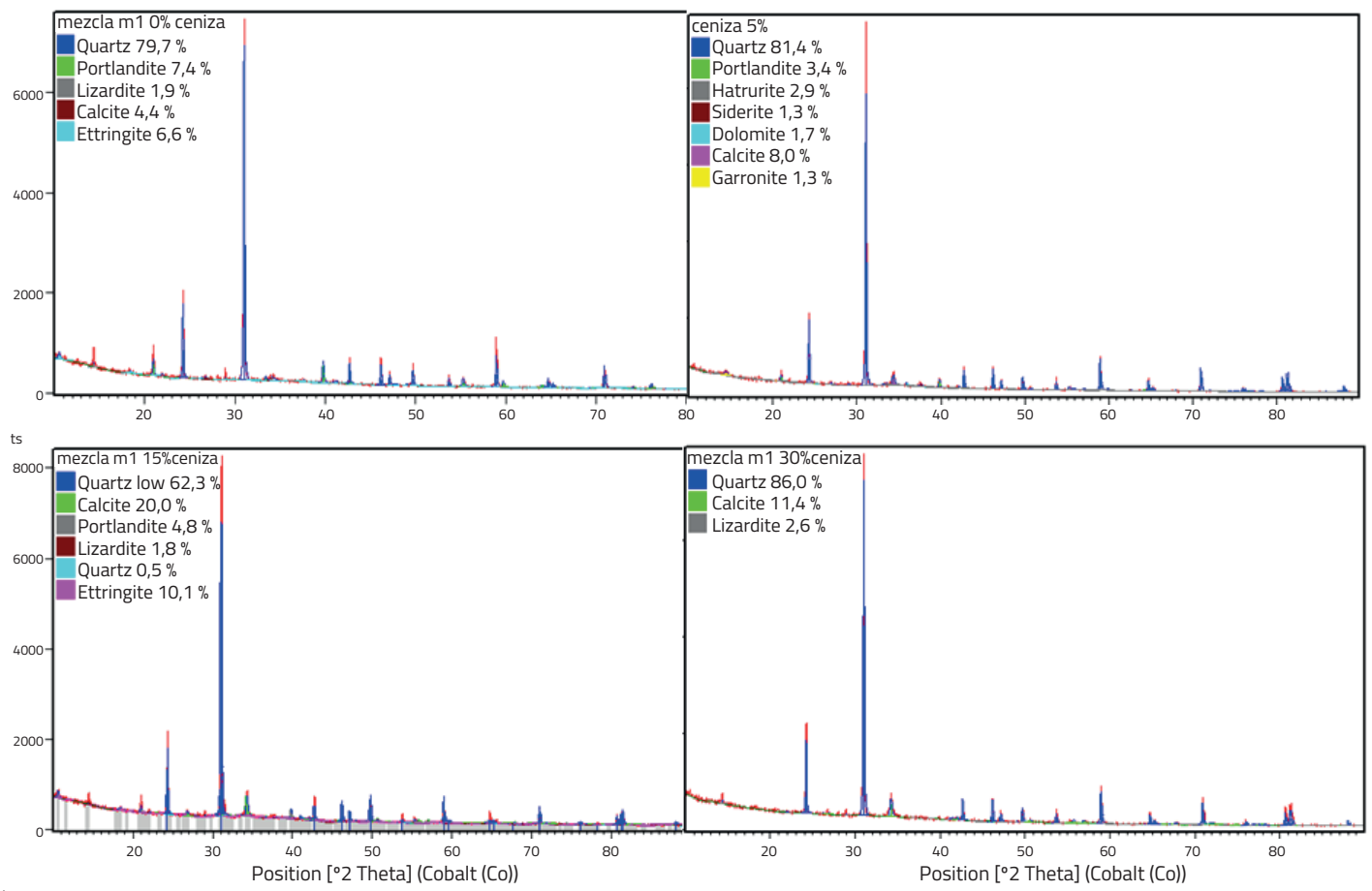

Figura 13. DRX muestras patrón y sustituciones al 5\%, 15\% y 30\%.

Fuente: Elaboración propia. 
TambiénserealizóanálisisdeMEBalasmuestras, de tal forma que se tiene para las sustituciones del $0 \%, 5 \%, 15 \%$ y $30 \%$. En este análisis se pone énfasis a la morfología encontrada en cada muestra, la cual inicialmente es una morfología amorfa para la muestra con sustitución del 0\% y se comienzan a ver unas superficies laminares a medida que el porcentaje de sustitución es más alto (Figura 14).

Teniendo en cuenta que para los concretos hidráulicos el ensayo más representativo es el de resistencia a flexión para comprobar su módulo de rotura, se realizó un análisis estadístico de los resultados obtenidos, tomando en cuenta que se está evaluando el efecto que tiene modificar la composición del concreto (agua + cemento + agregado grueso + agregado fino) sustituyendo en $0 \%, 5 \%, 15 \%$ y $30 \%$ la cantidad de cemento en la mezcla con ceniza de cascarilla del arroz, obtenida por altas temperaturas.
Se prueban 3 viguetas del tipo de cemento M1 (1), las concentraciones al $0 \%, 5 \%, 15 \%$ y $30 \%$ (4) a los 28 días de curado (1). $(3 \times 1 \times 4 \times 1)$ $=12$, con estos datos se realiza el análisis de varianza (Tabla 8).

La tabla ANOVA (Tabla 8) descompone la variabilidad de Módulo de rotura ( $\mathrm{MPa}$ ) en contribuciones debidasavariosfactores. Puesto que se ha escogido la suma de cuadrados tipo III (por omisión), la contribución de cada factor se mide eliminando los efectos de los demás factores. Los valores-P prueban la significancia estadística de cada uno de los factores. Puesto que un valor-P es menor que 0,05 , este factor tiene un efecto estadísticamente significativo sobre Módulo de rotura ( $\mathrm{MPa}$ ) con un 95,0\% de nivel de confianza.

Contraste de Hipótesis Tipo de cemento (M1)
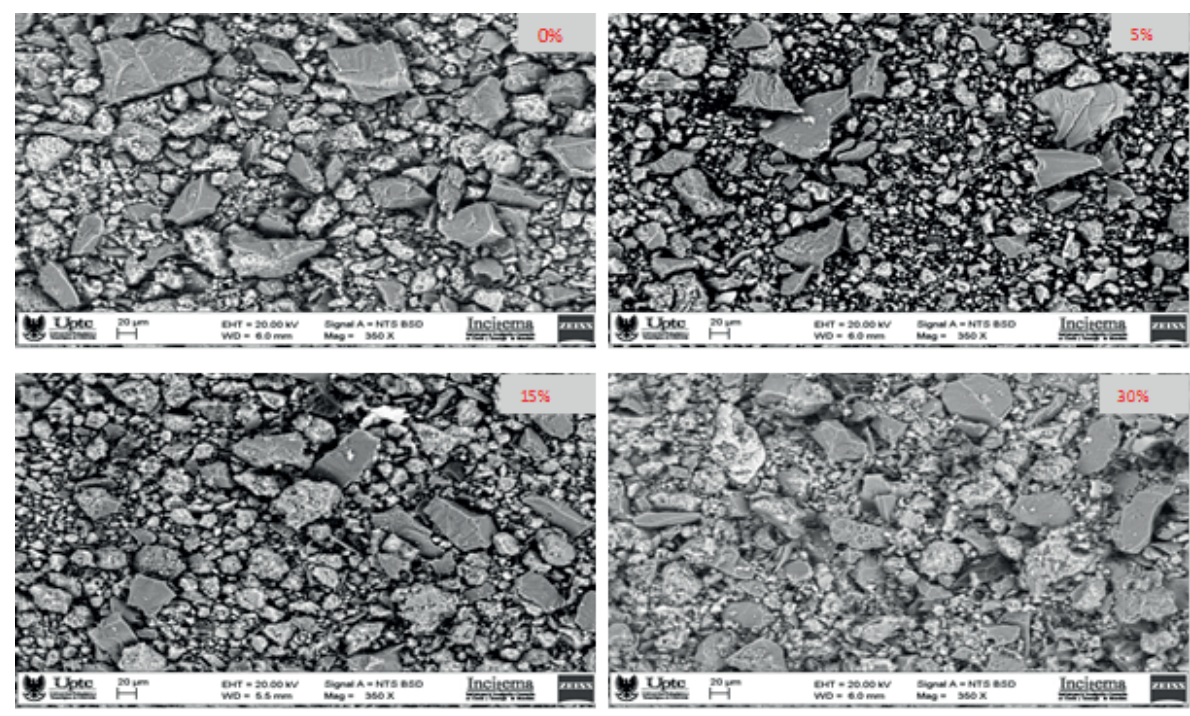

Figura 14. MEB muestras patrón y sustituciones al 5\%, 15\% y 30\%.

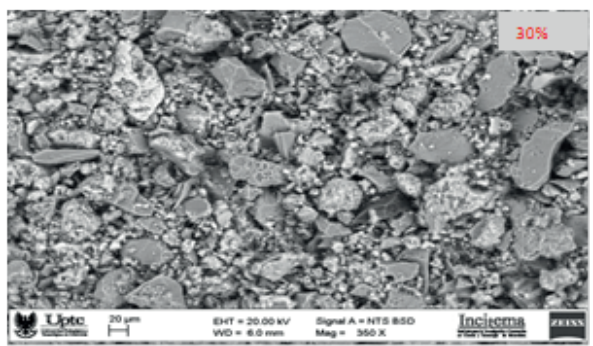

Fuente: Elaboración propia. 
Tabla 8. Análisis de Varianza para Módulo de rotura (MPa) - Suma de Cuadrados Tipo III

\begin{tabular}{|c|c|c|c|c|c|}
\hline Fuente & $\begin{array}{c}\text { Suma de } \\
\text { Cuadrados }\end{array}$ & GI & Cuadrado Medio & Razón-F & Valor-P \\
\hline A:Concentracion M1 & 21,391 & 3 & 7,13034 & 1160,98 & 0,0000 \\
\hline RESIDUOS & 0,0491333 & 8 & 0,00614167 & & \\
\hline TOTAL (CORREGIDO) & 21,4402 & 11 & & & \\
\hline
\end{tabular}

Todas las razones-F se basan en el cuadrado medio del error residual.

Fuente: Elaboración propia.

La concentración de ceniza en el concreto no influye en los valores de Módulo de rotura (MPa):

i. Se plantea la hipótesis nula y una hipótesis alternativa:

- $\mathrm{Ho}=$ Módulo de Rotura ${ }_{0 \%}=$ Módulo de Rotura $_{5 \%}=$ Módulo de Rotura $_{15 \%}=$ Módulo de Rotura ${ }_{30 \%}$

- $\mathrm{Ha}=$ Módulo de Rotura es distinto

ii. El nivel de significancia del análisis es del $95 \%: \alpha=0,05$

iii. Se establece estadístico de prueba mediante la Ecuación 2.

$F_{A}=\frac{\hat{S}_{A}^{2}}{\hat{S}_{R}^{2}}=\frac{m J \sum_{i=1}^{I}\left(\bar{Y}_{i . . .}-\bar{Y}_{\ldots}\right)^{2} / I-1}{\hat{S}_{R}^{2}} \rightarrow F_{I-1 ; I J(M-1)}$

iv. Como se puede observar en la tabla Anova (Tabla 8), como $F_{A}>F_{0.95,3,8,} ; F_{A}=1160.98$ $>\mathrm{F}_{0.95,3,8}=4.066$, se rechaza $\mathrm{H}_{0}$ con una confianza del $95 \%$, entonces se rechaza la hipótesis de que los módulos de rotura son similares.

Se realizó un modelamiento de la resistencia a la flexión vs la concentración de ceniza para la mezcla elaborada con cemento M1 (Figura 15). Analizando la propiedad mecánica de la resistencia a la flexión para evaluar el concreto hidráulico, y teniendo en cuenta la resistencia de diseño teórico de $4.21 \mathrm{MPa}$, tenemos que: las sustituciones del $15 \%$ y $30 \%$ de cemento por ceniza de la cascarilla del arroz son desfavorables porque la resistencia está por debajo de la requerida, pero para la sustitución del $5 \%$ presenta un beneficio porque incrementa su resistencia en un $11.4 \%$ (0.48 $\mathrm{MPa}$ ) con respecto a la teórica y un $9.8 \%$ (0.42 $\mathrm{MPa}$ ) con respecto a la muestra patrón.

Realizando una comparación de los resultados obtenidos en la Difracción de Rayos X (DRX) de la mezcla de concreto $M 1$ patrón y la mezcla de concreto M1 con sustitución del 5\% (Figura 13) para tratar de entender por qué la resistencia a la flexión aumenta de 4.27 MPa a 4.69 MPa respectivamente, se observa que al mezclarse el cemento con la ceniza de la cascarilla del arroz se genera un aumento en los silicatos de calcio, importantes en las propiedades cementantes del concreto, sin embargo se tendrá que revisar más a fondo qué estructuras internas se están formando y la actividad puzolánica de la ceniza para reforzar esta teoría. 

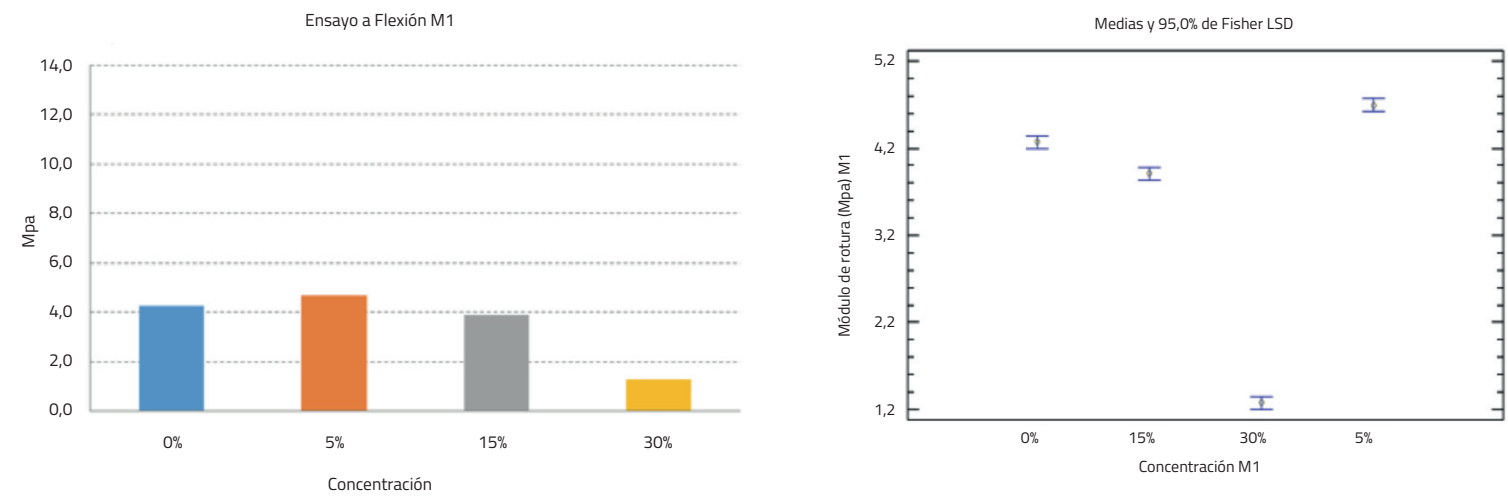

Figura 15. Comportamiento de la resistencia promedio a flexión. La resistencia más baja se da con mezcla al 30\%.

Fuente: Elaboración propia.

La ceniza de la cascarilla del arroz es un material puzolánico y altamente reactivo como se ha demostrado en otras investigaciones [8, 14-15, 23], que al mezclarse con el cemento afectan las reacciones químicas en la fase de hidratación mejorando la resistencia del concreto.

\section{CONCLUSIONES}

El proceso de incineración a altas temperaturas en este caso $+/-800{ }^{\circ} \mathrm{C}$ de la cascarilla del arroz garantiza la eliminación de los residuos orgánicos que alteran la composición y el comportamiento mecánico del concreto hidráulico; la ceniza obtenida muestra una composición con alto contenido de óxido silicio (91.4\%) de acuerdo al análisis de DRX, lo cual corrobora los datos encontrados en otras investigaciones.

La resistencia de diseño a flexión para la mezcla con cemento Holcim M1 Concretera
(4.21MPa) se cumplió para las muestras patrón (4.27MPa) y las muestras con sustitución del $5 \%$ (4.69MPa), por lo tanto hay factibilidad técnica de utilizar la ceniza de la cascarilla del arroz como reemplazo de cemento en la producción de concreto hidráulico, aunque las muestras con sustitución de $15 \%$ y $30 \%$ no hayan cumplido.

Las muestras con sustitución del 5\% presentaron un comportamiento mecánico superior a las muestras patrón en los ensayos de resistencia a la compresión, tracción indirecta y flexión, por lo tanto se evidencia el aporte en la resistencia de la ceniza de cascarilla del arroz, sin embargo para los porcentajes de sustitución del 15\% y $30 \%$ se genera una pérdida significativa de resistencia y no pueden ser usados para la fabricación de concreto hidráulico para pavimento rígido.

Este tipo de investigación puede servir de referente para dar solución a la problemática 
de los molinos de arroz que no tienen establecida una disposición final de la cascarilla del arroz y generarle un valor agregado a esta, adicionalmente se estaría produciendo un material que sería complemento del cemento y de esta forma reducir en algún grado el alto impacto ambiental que tiene la fabricación del mismo.

\section{AGRADECIMIENTOS}

Este trabajo fue financiado por la Dirección de Investigaciones (DIN) con radicado SGI-1783 de la Universidad Pedagógica y Tecnológica de Colombia. Además, agradecer al Instituto para la Investigación y la Innovación en Ciencia y Tecnología de Materiales - INCITEMA, por sus análisis de DRX y MEB a los materiales, igualmente a la Fundación Universitaria Internacional del Trópico Americano por el préstamo de sus laboratorios de suelos y pavimentos.

\section{REFERENCIAS}

[1] Rendón, D., Ospina, M., \& Mejía, R. (2009). Evaluación de la sílice obtenida de un subproducto industrial en pastas y morteros de cemento portland. Revista informador técnico, pp. 20-26.

[2] Chao-Lung, H., Anh-Tuan, B. \& ChunTsun, C. (2011). Effect of rice husk ash on the strength and durability characteristics of concrete. Construction and Building Materials, 25, pp. 3768-3772. doi:10.1016/j.conbuildmat.2011.04.009

[3] Ferraro, R. \& Nanni, A. (2012). Effect of off-white rice husk ash on strength, porosity, conductivity and corrosion resistance of white concrete. Construction and Building Materials, 31, pp. 220-225. doi:10.1016/j.conbuildmat.2011.12.010

[4] Vásquez, R. \& Bach, P. (2000). Las cenizas de cáscara de arroz; adición puzolánica en cemento y concreto. Universidad de Piura. Piura, Perú.

[5] Jamil, M., Kaihsentes, A., Raman, S. \& Zain, M. (2013). Pozzolanic contribution of rice husk ash in cementitious system. Construction and Building Materials, 47, pp. 388-593. doi:10.1016/j. conbuildmat.2013.05.088

[6] Fuentes, N., Fragozo, O. \& Viscaino, L. (2015). Residuos agroindustriales como adiciones en la elaboración de bloques de concreto no estructural. Ciencia $e$ Ingeniería Neogranadina, 25, pp. 99-116. doi:10.18359/rcin. 1434

[7] Mattey, P., Robayo, R., Díaz, J., Delvasto, S. \& Monzó, J. (2015). Influencia del mezclado en dos etapas en la fabricación de ladrillos de mampostería con ceniza de cascarilla de arroz como agregado fino. Revista Colombiana de Materiales, 5, pp. 242-249.

[8] Águila, I. \& Sosa, M. (2008). Evaluación físico químico de cenizas de cascarilla de arroz, bagazo de caña y hoja de maíz y su influencia en mezclas de mortero, como materiales puzolánicos. Revista de la Facultad de Ingeniería Universidad Central de Venezuela, 23, pp. 55-66.

[9] Serrano, T., Borrachero, V., Monzó, J. \& Payá, J. (2012). Morteros aligerados con cascarilla de arroz: diseño de mezclas y evaluación de propiedades. Revista DYNA, Facultad de Minas, 175, pp. 128-136.

[10] Jiménez, V., León, F., Montes, P., Gaona, C. \& Chacón, N. (2013). Influence of sugarcane bagasse ash and fly ash on the rheological behavior of cement pastes and mortars. Construction and Building Materials, 40, pp. 691-701. doi:10.1016/j. conbuildmat.2012.11.023 
[11] Xu, W., Lo, Y., Ouyang, D., Memon, S., Xing, F., Wang, W. \& Yuan, X. (2015). Effect of rice husk ash fineness on porosity and hydration reaction of blended cement paste. Construction and Building Materials, 89, pp. 90-101. doi:10.1016/j. conbuildmat.2015.04.030

[12] Hesami, S., Ahmadi, S. \& Nematzadeh, M. (2014). Effects of rice husk ash and fiber on mechanical properties of pervious concrete pavement. Construction and Building Materials, 53, pp. 680-691. doi:10.1016/j.conbuildmat.2013.11.070

[13] Santaella, L. \& Salamanca, R. (2004). Comportamiento del concreto con bajos porcentajes de ceniza volante (Termopaipa IV) y agua constante. Ciencia e Ingeniería Neogranadina, 14, pp. 14-19. doi:10.18359/rcin.1264

[14] Satish, V., Vikrant, V., \& Kavita, K. (2013). Combine effect of rice husk ash and fly ash on Concrete by $30 \%$ cement replacement. Procedia Engineering, 51, pp. 35-44. doi:10.1016/j.proeng.2013.01.009

[15] Salas, A., Mejía, R., \& Delvasto, S. (2013). Developing high-performance concrete incorporating highly-reactive rice husk ash. Ingeniería e Investigación, 33, pp. 49-55.

[16] Chabanes, M., Bénezet, J., Clerc, L. \& Garcia E. (2014). Use of raw rice husk as natural aggregate in a lightweight insulating concrete: An innovative application. Construction and Building Materials, 70, pp. 428-438. doi:10.1016/j. conbuildmat.2014.07.025

[17] DANE. (20 de febrero de 2015). Encuesta nacional de arroz mecanizado. En: http:// www.dane.gov.co/files/investigaciones/ boletines/arroz/bol_arroz_Ilsem14.pdf? (15 de abril de 2015).

[18] Arcos, C., Macías, D., \& Rodríguez, J. (2007). Husk of rice as source of SiO2.
Revista Facultad de Ingeniería Universidad de Antioquia, 41, pp. 7-20.

[19] Martínez, J., Pineda, T., López, J. \& Betancur, M. (2010). Experimentos de combustión con cascarilla de arroz en lecho fluidizado para la producción de ceniza rica en sílice. Revista Facultad de Ingeniería Universidad de Antioquia, 51, pp. 97-104.

[20] Zerbino, R., Giaccio, G. \& Isaia, G. (2011). Concrete incorporating rice-husk ash without processing. Construction and Building Materials, 25, pp. 371-378. doi:10.1016/j.conbuildmat.2010.06.016

[21] Rajamma, R., Ball, R., Tarelho, L., Allen, G., Labrincha, J. \& Ferreira, V. (2009). Characterisation and use of biomass fly ash in cement-based materials. Journal of Hazardous Materials, 172, pp. 1049-1060. doi:10.1016/j.jhazmat.2009.07.109

[22] Mejía, J., Mejía de Gutiérrez, R., \& Puertas, F. (2013). Rice husk ash as a source of silica in alkali-activated fly ash and granulated blast furnace slag systems. Materiales de Construcción, 63, pp. 61-375. doi:10.3989/mc.2013.04712

[23] Salas, A., Mejía, R., \& Delvasto, S. (2009). Comparison of two processes for treating rice husk ash for use high performance concrete. Cement and Concrete Research, 39, pp. 773-778. doi:10.1016/j.cemcon res.2009.05.006

[24] Rivera, G. (2013) Concreto simple. Civilgeeks. En: http://civilgeeks.com/2013/ 08/28/libro-de-tecnologia-del-concreto$\mathrm{y}$-mortero-ing-gerardo-a-rivera-l/. 